\title{
Android Application for Visually Impaired People using Text-To-Speech
}

\author{
Asst. Prof. A. R. Sonawane ${ }^{1}$, Abhijeet Wankhede ${ }^{1}$, Komal Rasane ${ }^{1}$, Vivek Baraskaev ${ }^{1}$, Ganesh Borde ${ }^{1}$ \\ NBNSSOE, Pune, India ${ }^{1}$
}

\begin{abstract}
As the use of mobile devices is expanding and affecting various aspects of human life, the number and smartphone users is dramatically increasing. Consequently, the robustness of interaction between smartphone and human is essential for better system performance. By using the speech synthesizer technology for the android, the application presents the modality of text-to-speech responses on android device. In user mobile device they provided Location Based Services for set of services which originate from the geographic location. Using these services it is possible for the users to finding and locate other area, vehicles, and resources and also to provide location- services, in addition to tracking their own location. The request for location can originate by mobile device. When the mobile device is at any particular location it is possible to automatically trigger Location Based Services. These services can also helpful in the user's mobile device itself in order to satisfy location-based queries like finding areas of interest, checking traffic conditions, finding our friends, or vehicles, resources, machines and emergency requests. In this paper we will discuss how to implement these location based services in Android after giving introduction to Android and its constituents.
\end{abstract}

Keywords: Language learning, text -to- speech, speech synthesis.

\section{INTRODUCTION}

Speech application can be defined as communication between the user and the computer in more natural way or language. As people find speaking naturally is easy, it is the most advantageous to incorporate speech into any natural language processing software. Conversational dialog is a verbal action and it can takes place turn by turn between human and computer.

Feedback and acknowledgement to indicate understanding. The field of Artificial Intelligence (AI) and the idea of a machine learning with humans are as old as the field of Computer Science.

Speech application should be work on an understanding of the different ways that people use language to communicate. Nowadays people use texting and IVR (Interactive voice response) to communicate with the computers via cell phone. IVR system can be used by with the speech recognition. To order or book something with this kind of application, it follows the exact conversational dialog. IVR is pre-recorded audio to direct user how to proceed. With the use of speech recognizer and speech synthesizer, the applications based on IVR can be deployed to automobile systems for hands-free operation. Where grammar is predefined, most of the time IVR based application can be used for transactional dialog, and user is bound to say restricted quarries.

Despite of the advanced AI tools available, the question always remained for how to translate a semantic structure into computer queries or commands that can re-use existing commercial applications and databases that are proprietary to a specific business. Furthermore, such AI suited languages are difficult to use and to grasp with the mind. In the latter years software developers have been forced to abandon these languages that are better suited for natural language and option to develop specific dialog flows from scratch using Java, VoiceXML, and now C++. The dialog is designed for the specific application, but it tends to limit the user to specify commands, due to the task-oriented nature of these languages. Although these languages have Object Oriented capabilities they are still very much task oriented.

\section{ALGORITHM EXPLANATION}

\section{A. NATURAL LANGUAGE PROCESSING}

Natural language processing (NLP) is a field of computer science, artificial intelligence, concerned with the interactions between computers andhuman (natural) languages. As such, NLP can be used in the area of human- computer interaction. Many challenges in NLP involve: enabling computers to derive meaning natural language input; and others involve natural language generation.

There are so many Natural processing algorithms among which we will be using the TTS i.e. Text to Speech algorithm, which is explained as follows:

\section{B.TEXT TO SPEECH}

A text-to-speech system (or "engine") is composed of two parts: a front-end and aback-end. The front-end has two major tasks. First, it converts raw textcontaining symbols like numbers and similarly into the equivalent of written- 
out words. This process is often called text To develop the Application with Natural Language normalization, pre-processing, or tokenization. The front- Processing and Text-to-Speech, we have used the Clientend then assigns phonetic transcriptions to each word, then Server Architecture approach. As the goal of the divides text into prosodic units, like phrases, clauses, application is to provide text-to-speech functionality on and sentences etc. The process of assigning phonetic mobile device, it uses Android operating system device as transcriptions to words is called text-to-phoneme or client. It can be any model of the phone using Android 2.2 grapheme-to-phoneme conversion. Phonetic transcriptions or above version. and information together make up the symbolic representation that is output by the front-end phase. The back-end phase-often referred to as the synthesizerthen converts the symbolic representation or conversion into sound. In certain systems, this part includes the computation of the target prosody(pitch contour, phoneme durations),[4] which is then imposed on the output speech.

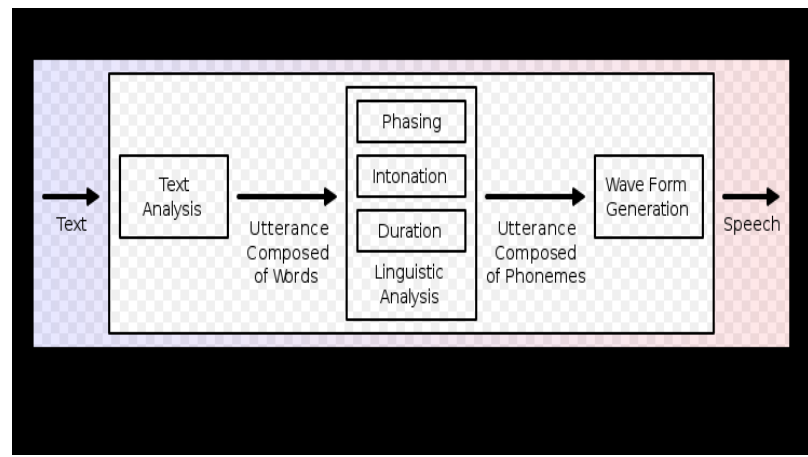

Figure1:Working of NLP

\section{BLOCK DIAGRAM\& EXPLANATION}

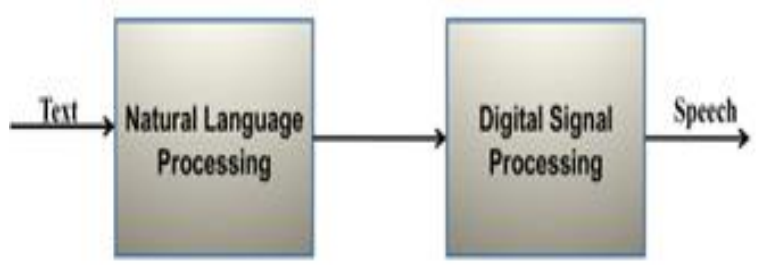

Figure 2: Text-Speech Synthesizer

A simple text is process by Natural language processing software with linguistic knowledge and some logical inferences. Then the text goes to make some phonetic transcription with desired intonation and rhymes. Then it passes through the Digital Signal processing to transform that symbolic information into speech with the help of mathematical models, algorithms and computations.

\section{A. Architecture}

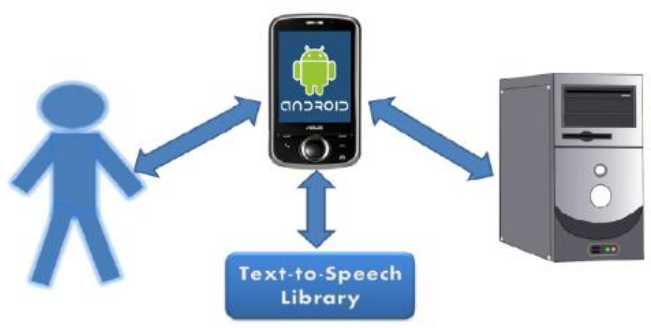

Figure 3: System Architecture
On the server side, The Natural Language Parser is developed sin Java. The grammars and dictionaries used for the application are in the .txt format packaged in 'Language' directory of the application package.

User can connect to the server from the android phone by giving the host name and the port number of the server. User can speak or type the question to the device and then it connects to the server to parse the speech and process it using natural language processing.

The server communicates to the android client and gives the response in text format. Android client is using Textto-speech library to process the text into speech. Finally user can get the response of the question asked in speech format on android device.

\section{CONCLUSION}

The Natural Language Processing tool with text-to-speech looks through dictionaries and grammars to gather relevant response of user request believe that the application is developed using the concepts and design of natural language processing when deployed, helps the end user to speak more naturally for the reservation process.

This project uses primarily the android device, with textto-speech library supported for android operating system. Second, this application gives the appropriate responses based on the natural language parser and understanding tool to flow the user communication with android smartphone more natural way.

Further the solution implemented during this project is scalable, portable, can be deployed to any android device. Location Based Services are those services which provide both information and entertainment and are accessible with mobile devices through the mobile network.

They utilize the ability to make use of the geographical position of the mobile device. They can utilize multiple technologies such as the GPS satellite network, Wi-Fi networks and other technologies.

\section{ACKNOWLEDGMENT}

The Acknowledgment of our project is to helpful for impaired people.by using this features they finding their way easily. It can used for security purpose. Security pattern was good for that. 


\section{REFERENCES}

[1] A. Turing, "Computing Machinery and Intelligence", Mind 49, 1950, pp. 433-460.

[2] "Designing Effective Speech Application", JavaTM Speech API Programmer's Guide, Sun Microsystems, Inc.

[3] C.Bajorek, "The state of IVR navigation technology", Computer Telephony Magazine, New York, NY, Volume 8, September 2000.

[4] J. A. Jocko, A. Sears, "The human -computer interaction handbook: fundamentals, evolving technologies, and emerging" New Jersey: Lawrence Erlbaum Associates, 2003, pp. 712-750.

[5] T.Dutoit, "An Introduction to Text-to-Speech Synthesis." TTS Research team, TCTS Lab, pp. 2-6.

[6] B.Manaris. "Natural Language Processing: A human-Computer Interaction Perspective", University of Southwestern Louisianan, Louisiana.

[7] Chomsky, N.. "Remarks on Nominalization." In Jacobs \& P. Rosenblum, eds., Readings in English, 1970.

[8] Jackendoff, R. 'Foundations of Language.' Oxford University Press,New York, NY. 2002

[9] Jackendoff, R. 'Semantics and Cognition.' The MITPress, Cambridge,MA. 1983.

[10] NuGramPlatform,"http://nugram.nuecho.com/product_app/welcom e",nu Echo Inc., 2003-2011. 